\title{
Neurofilament Redistribution in Transected Nerves: Evidence for Bidirectional Transport of Neurofilaments
}

\author{
Jonathan D. Glass' ${ }^{1}$ and John W. Griffin ${ }^{2}$ \\ Departments of 'Neurology and Pathology (Neuropathology) and 2Neurology and Neuroscience, Johns Hopkins University \\ School of Medicine, Baltimore, Maryland 21205
}

\begin{abstract}
Nerve fibers of the C57BL/6/Ola mouse exhibit very slow Wallerian degeneration following axotomy, thus allowing prolonged observation of mammalian axons separated from their cell bodies. The present study utilized teased-fiber preparations, silver histochemistry, immunocytochemistry, and electron microscopy to examine the distribution of axonal components in the distal stumps of axotomized sciatic nerves in C57BL/6/Ola mice. In examining nerve segments at varying intervals after nerve transection, we found no evidence of proximal-to-distal "emptying out" of the cytoskeleton, as would be predicted if the cytoskeleton in these transected nerves were undergoing anterograde transport as an assembled structure. Instead, we observed a gradual redistribution of cytoskeletal constituents over time, dominated by the progressive accumulation of neurofilaments at the severed ends of axons. In particular, there were massive accumulations at the proximal ends of the distal stumps. These results strongly suggest that, at least in transected nerve fibers, neurofilaments can be transported bidirectionally.
\end{abstract}

Neurofilaments, the major type of intermediate filaments in neurons, are the most prominent cytoskeletal elements in the axons of large nerve fibers. They are synthesized in the cell body of the neuron and are transported anterogradely down the axon within the slow component of axonal transport, at rates of $0.2-$ $2 \mathrm{~mm} / \mathrm{d}$ (Hoffman and Lasek, 1975). The macromolecular form in which neurofilament proteins are transported and the proportion undergoing transport at any one time are currently controversial issues. A widely held view is that neurofilaments are transported from the cell body to the axon as part of an assembled cytoskeleton (Lasek and Hoffman, 1976; Lasek et al., 1984). In this model, the cytoskeleton moves continuously from proximal to distal until it is ultimately degraded at the terminal by intrinsic axonal proteases. This model predicts that, in nongrowing axons, degradation of neurofilaments at the terminal must equal synthesis at the cell body. An alternative view is that the cytoskeleton is largely stationary (Nixon and Logvinenko, 1986; Hollenbeck, 1990) and that neurofilaments are

\footnotetext{
Received Mar. 4, 1991; reviscd Apr. 30, 1991; acceptcd May 3, 1991.

We thank J. Coram, N. Crouse, C. Rubright, M. Shagogue, and A. Stocks for technical assistance, T. Crawford for assistance with morphometry, D. Price and P. Hoffman for discussion, and R. Graham for manuscript preparation. This work was supported by NIH Grants NS 07179 and PO1 22849.

Correspondence should be addressed to Jonathan D. Glass, Departments of Neurology and Pathology (Neuropathology), Johns Hopkins University School of Medicine, Pathology 622a, 600 North Wolfe Street, Baltimore, MD 21205.

Copyright (C) 1991 Society for Neuroscience $0270-6474 / 91 / 113146-09 \$ 05.00 / 0$
}

replenished by the transport of individual neurofilaments or small bundles of neurofilaments, of particles containing neurofilament proteins (Weisenberg et al., 1987), or of subunits that are inserted into the cytoskeleton along its length (Ochs, 1982; Ochs et al., 1989). The last three models imply that the proportion of the total content of neurofilament protein undergoing transport in the axon at any one time could be low. In all of these models, transport of neurofilaments has been postulated to occur predominantly, if not exclusively, in the anterograde direction.

Axonal transport can be studied by following the redistribution of specific axonal constituents in nerves isolated from their source of newly synthesized proteins in the nerve cell bodies. For example, in nerve segments that were isolated from the rest of the nerve by ligations, rapidly transported markers accumulated at both the proximal and distal ends of the segment, providing some of the early evidence for the existence of fast bidirectional transport (Ranish and Ochs, 1972; Brimijoin, 1975; Griffin et al., 1976; Brimijoin and Wiermaa, 1977; Lubinska and Niemierko, 1977). These transport systems carry membrane-bound vesicles at rates of $\sim 200-400 \mathrm{~mm} / \mathrm{d}$, so that substantial redistribution of axonal constituents can occur in the interval prior to the axonal degeneration that is induced by the interruption of the axon. For molecules that are transported in the slow component, however, this approach is less useful, except in nonmammalian systems where axons survive for prolonged periods of time after being separated from their cell bodies. For example, transport studies in goldfish optic nerves (Alpert et al., 1980) and garfish olfactory nerves (Cancalon, 1982) have shown that slow axonal transport continues within the distal stump. In the garfish, morphologic studies showed that the cytoskeleton of the transected distal stump "empties out" from proximal to distal at the rate of slow axonal transport (Cancalon, 1982). This observation supported models in which the assembled cytoskeleton is transported intact. In mammalian nerve fibers, this approach has not been utilized because the process of Wallerian degeneration is relatively rapid compared to the rate of slow transport. For example, in the peripheral nerves of young rodents most of the axons of the distal stump are reduced to granular debris within $24-48 \mathrm{hr}$; neurofilaments moving at their normal rates of transport would be expected to move only $1-2 \mathrm{~mm}$ in this interval, precluding analysis of their redistribution.

The C57BL/6/Ola mouse, a strain in which peripheral nerves undergo very slow Wallerian degeneration following axotomy (Lunn et al., 1989; Perry et al., 1990; Brown et al., 1991), allows prolonged observation of the mammalian axon and its cytoskeleton in isolation from its cell body. We examined the changes 
in axons during prolonged survival following sciatic nerve interruption in the C57BL/6/Ola mouse. We observed a dissociation and redistribution of cytoskeletal components over time, which resulted in regional concentrations of neurofilaments and microtubules along the axon, and giant accumulations of neurofilaments at the ends of isolated stumps. These data indicate that although transport continues in the distal stump, there is no bulk movement of the cytoskeleton from proximal to distal. Rather, because neurofilaments accumulated at the proximal end of the isolated segment, we suggest that cytoskeletal transport in these stumps includes retrograde as well as anterograde transport of neurofilaments.

\section{Materials and Methods}

C57BL/6/Ola mice were obtained from a breeding colony maintained at the Johns Hopkins University School of Medicine. Sixty mice, male and female, aged 12-16 weeks, were used. C57BL/6 mice of the same age were obtained commercially (Harlan Sprague Dawley, Frederick, $\mathrm{MD}$ ) and used as controls. Surgery was performed under methoxyflurane anesthesia according to one of two protocols: (1) sciatic nerves were transected with fine scissors at a single site at the level of the sciatic notch; or (2) sciatic nerve transections were performed at two siteswithin the pelvis and at the sciatic notch ("double axotomy"; Fig. 1). The double axotomy protocol was used to study segments of nerves isolated from both their cell bodies and their terminals. To address the issue of nerve retraction following transection, another group of animals underwent sciatic nerve crush, which interrupts individual nerve fibers but keeps the connective tissue structure of the nerve intact.

Animals were killed under deep anesthesia either by perfusion through the ascending aorta or by exsanguination following cardiac transection. The tissue fixatives were $4 \%$ buffered paraformaldehyde ( $\mathrm{pH} 7.2$ ) or $5 \%$ buffered glutaraldehyde (pH 7.2). Some nerves were postfixed in osmium tetroxide, embedded in Epon, cut at $1 \mu \mathrm{m}$, and stained with toluidine blue for light microscopy. Thin sections for electron microscopy were stained with uranyl acetate and lead citrate and examined with a Hitachi 600 electron microscope. Other nerves were embedded in paraffin and cut longitudinally at $10 \mu \mathrm{m}$. Some sections were stained by standard reduced silver techniques for axons. Others were stained immunocytochemically for neurofilaments with antibodies SMI 31 or SMI 32 (Sternberger Monoclonals, Baltimore, MD; concentration, $1: 1000$ ) by the peroxidase/antiperoxidase technique (Sternberger, 1979). Teased fibers were prepared by staining paraformaldehyde-fixed nerve bundles with reduced silver, infiltrating the bundles with glycerol, and teasing apart individual nerve fibers with fine needles.

Neurofilament densities were calculated by counting the numbers of neurofilaments within designated areas in transverse sections, from electron micrographs enlarged 100,000 times. All of the neurofilament profiles were counted within $25 \mathrm{~cm}^{2}$ boxed areas on $8^{\prime \prime} \times 10^{\prime \prime}$ electron micrographs. Multiple areas in each axon were counted and averaged as the mean density of neurofilaments. The cross-sectional axonal areas were measured from $1 \mu \mathrm{m}$ plastic sections by using a computer-based digitizing system (BioQuant, Nashville, TN). The lengths of axonal swellings were measured from silver-stained and plastic-embedded longitudinal sections, by use of the same computerized system.

\section{Results}

\section{Course of Wallerian degeneration}

In axotomized nerves of normal C57BL/6 mice, no intact axons were demonstrable in distal stumps at $72 \mathrm{hr}$, and $>90 \%$ of axons were reduced to granular debris within $48 \mathrm{hr}$. At $7 \mathrm{~d}$, myelin clearance was well underway, with abundant myelin ovoids, infiltrating macrophages, and proliferated Schwann cells (Fig. $2 A$ ). In contrast, in the distal stumps of C57BL/6/Ola animals, axons did not show evidence of degeneration for at least $7 \mathrm{~d}$ (Fig. $2 B$ ), and many axons survived for up to $28 \mathrm{~d}$. Macrophages were seen in numbers proportional to the degree of degeneration. They were scarce within the first week except at the sites of transection, where macrophages phagocytosed the myelin debris

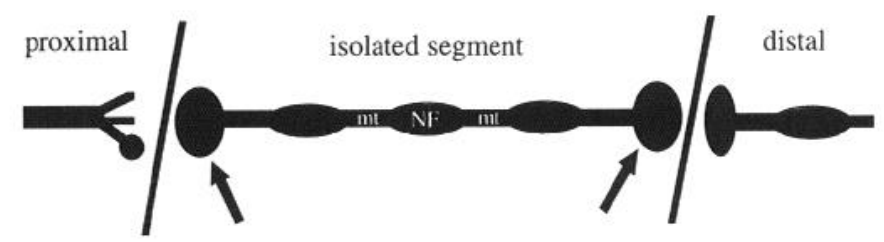

Figure 1. Diagram summarizing the "double axotomy" method and depicting the morphologic findings in transected axons in C57BL/6/Ola mice. Sciatic nerves were simultaneously transected at two levels using fine scissors, creating a segment isolated from both the cell bodies and terminals. In the isolated segment, giant neurofilamentous swellings were found at the proximal and distal ends of transected axons (arrows). In the midportion of the isolated segment, regions containing excessive neurofilaments $(\mathrm{NF})$ alternate with those containing few neurofilaments and high concentrations of microtubules $(m t)$. These findings were also present in the nerve distal to the distal stump, as depicted. Sprouting of axons was noted in the proximal stump but not in the isolated segment or most distal stump.

produced as a result of the local trauma. Even in these regions, the axons survived.

The rate of degeneration of sciatic nerves among C57BL/6/ Ola mice was generally constant. However, some fibers within the sciatic nerve of an individual mouse degenerated at faster rates than others. This variation in the rate of degeneration was observed among different fascicles. Within individual fascicles, fibers tended to degenerate at the same rate, as previously noted by Perry et al. (1990). More rapidly degenerating fascicles contained greater numbers of macrophages than adjacent, more preserved fascicles.

Unperturbed sciatic nerves of C57BL/6/Ola and C57BL/6 mice were morphologically identical. After axotomy, the proximal stumps of sciatic nerves in C57BL/6/Ola mice reacted in a similar fashion to those in C57BL/6 animals. Traumatic neuromas, consisting of abundant axonal sprouts ensheathed by Schwann cells and surrounded by fibroblasts and perineurial cells, formed progressively between several days and several weeks following axotomy. In contrast, the surviving distal stumps in C57BL/6/Ola mice showed no evidence of sprouting from either the proximal or distal (double axotomy) cut site. The axons of the distal stump underwent a gradual and progressive rearrangement of their cytoskeletal constituents, forming regional concentrations of neurofilaments and microtubules as described below.

\section{Cytoskeletal changes in midportions of fibers}

Accumulations of densely packed and maloriented neurofilaments developed over time throughout the axons in the distal stumps and isolated segments of the C57BL/6/Ola animals. These neurofilamentous swellings created two distinct pathological patterns. The first type of swelling was distributed throughout the midportion of the isolated segment, and the second was found at the site(s) of axotomy (see Fig. 1). The axonal changes in the midportion were best appreciated in longitudinal silverstained preparations (Fig. $3 A$ ). After several days, fibers took on a beaded appearance with alternating segments of enlargement and decrease in caliber. In teased fibers, it was clear that this alternating pattern occurred within single fibers. The areas of enlargement contained accumulations of densely packed and maloriented neurofilaments (neurofilamentous swellings; Fig. $3 B$ ), as evaluated by electron microscopy. These swellings, which stained with monoclonal antibodies SMI 31 and SMI 32 against 


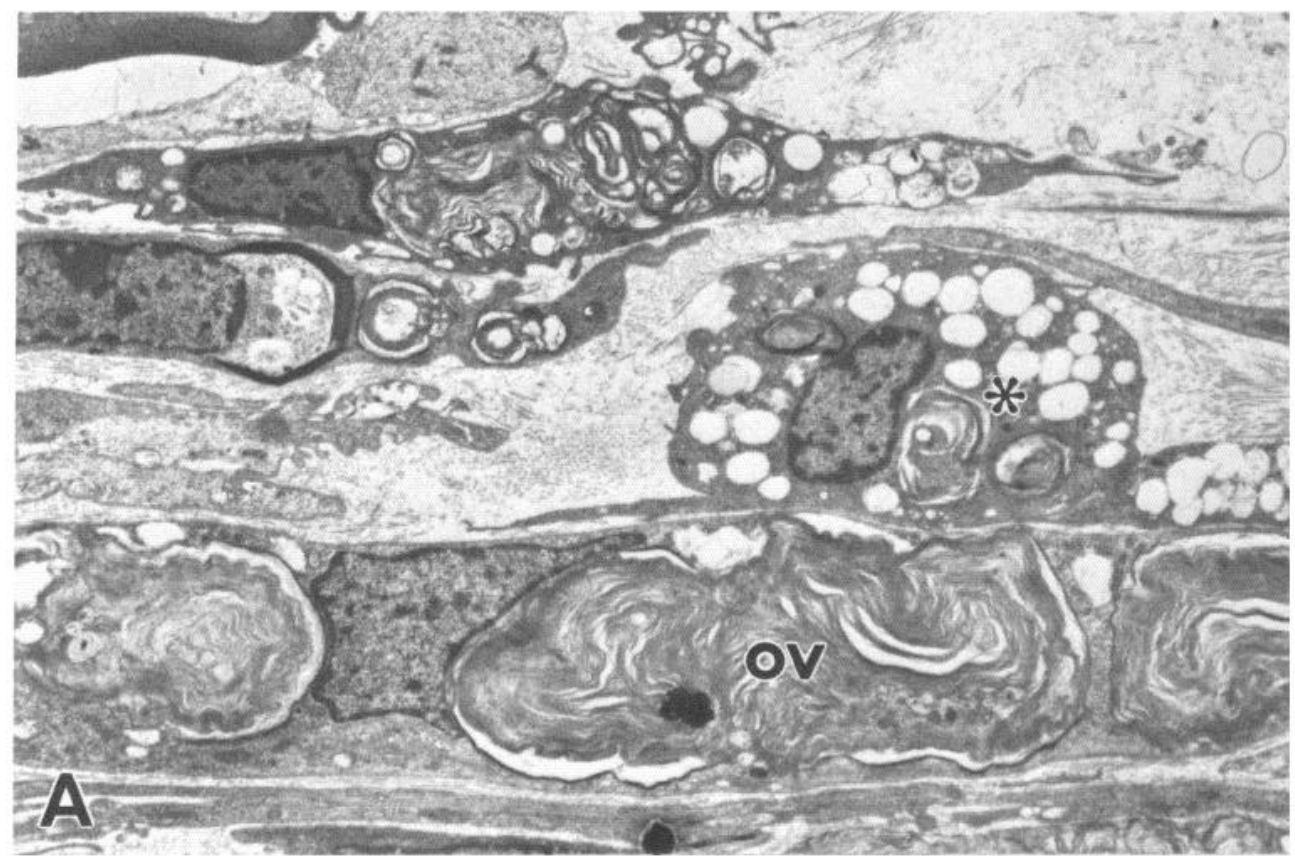

Figure 2. A, Longitudinal section through a region of the sciatic distal stump from a C57BL/6 mouse, $7 \mathrm{~d}$ after transection. No axons remain, myelin ovoids (ov) are abundant, and phagocytic macrophages (asterisk) are easily identified. Magnification, $4900 \times$. B, Similar section from a C57BL/6/Ola mouse, $7 \mathrm{~d}$ after transection. Note the normal appearance. $a$, axon; sc, Schwann cell. Stains, uranyl acetate and lead citrate. Magnification, $3120 \times$.

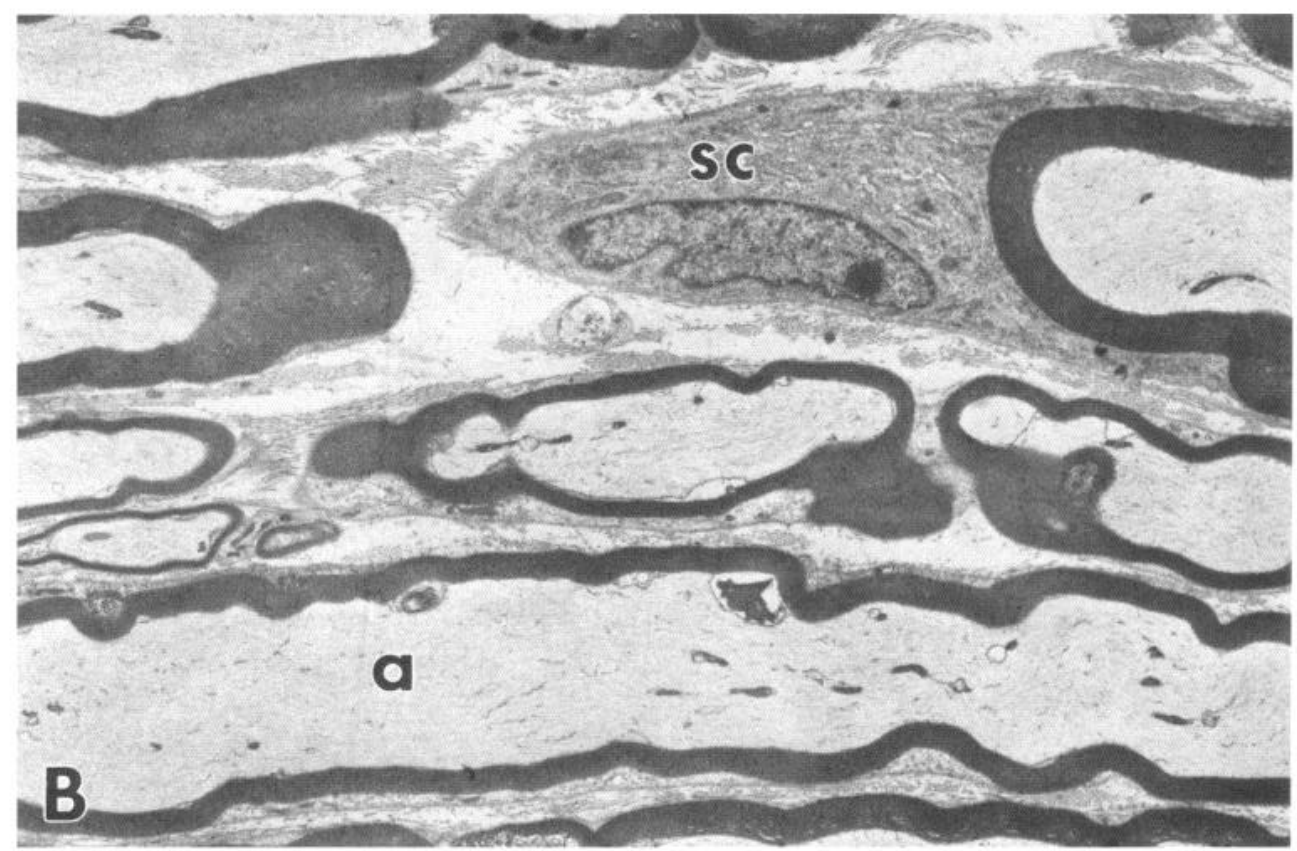

phosphorylated and nonphosphorylated epitopes of neurofilament, were found in both myelinated and unmyelinated fibers.

Microtubules were notably deficient in the regions of neurofilamentous swelling. In some instances, microtubules were concentrated within regions relatively devoid of neurofilaments. These segments appeared to have been reduced in caliber, as indicated by their relatively thick myelin sheaths (Fig. $3 \mathrm{C}$ ), and presumably correlated with the narrowed portions of the beaded fibers seen with silver stains.

\section{Cytoskeletal changes at cut ends}

Neurofilamentous swellings also developed at the proximal faces of distal stumps and at the proximal and distal faces of isolated segments ("double axotomy"; see Fig. 1). In longitudinal prep- arations, these swellings appeared at the ends of fibers as spherical or cylindrical enlargements that stained by silver histochemistry and by immunocytochemistry for phosphorylated and nonphosphorylated neurofilaments. Teased-fiber preparations were particularly informative because they demonstrated the relationship between the swellings and the ends of fibers (Fig. 4). These neurofilamentous swellings were first detected at 3-4 $\mathrm{d}$ following axotomy and continued to enlarge at least until 14 d. These swellings contained accumulations of densely packed and maloriented neurofilaments that were generally much larger than the neurofilamentous swellings occurring in the midportions of distal stumps. Again, few if any microtubules were present within the swellings. Myelinated axons containing large swellings often had thin or absent myelin sheaths (Fig. $5 B, D$ ), 

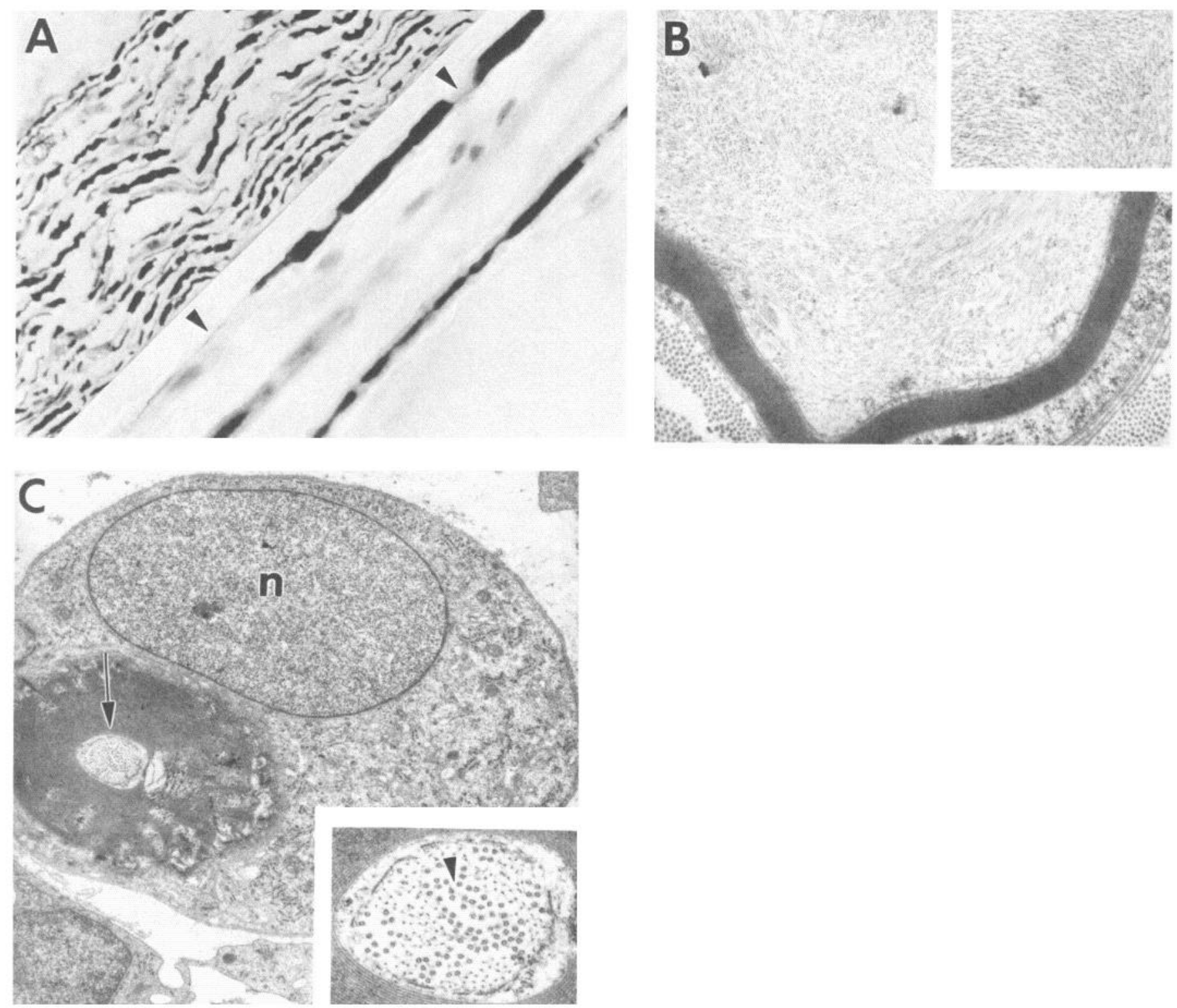

Figure 3. Axonal changes seen in the midportions of isolated segments of sciatic nerves of C57BL/6/Ola mice, $14 \mathrm{~d}$ following axotomy. A shows the beaded appearance of fibers in silver-stained paraffin section (top left; magnification, 80x) and teased fibers photographed under Nomarski optics (bottom right; magnification, 250×). Note that the atrophic regions in the teased fibers (arrowheads) are continuous with swollen regions. $B$, A swollen axonal region containing densely packed neurofilaments. Stain: lead citrate and uranyl acetate. Magnification, 16,400 $\times$. Inset, Detail of neurofilament density. Magnification, $19,900 \times . C$. Atrophic axonal regions show a disproportionately thick myelin sheath, indicating that the axon (arrow) has shrunk from a previously larger caliber. Magnification, $9900 \times$. Inset, This shrunken axon at the center of an internode contains few neurofilaments and high concentrations of microtubules (arrowhead). Magnification, 32,400×. N, Nucleus of Schwann cell.

reflecting the displacement of myelin lamellae from their usual termination site when swellings were present at the paranodes, as observed in other conditions characterized by axonal swelling (Jones and Cavanagh, 1983; Stanley et al., 1985).

To estimate the number of neurofilaments accumulating in these giant axonal swellings, the swollen axons at the most proximal segments of distal stumps were compared with the largest myelinated axons in unperturbed sciatic nerves of C57BL/6/ Ola mice in $1 \mu \mathrm{m}$ plastic cross sections and electron micrographs (Fig. 5). The mean cross-sectional axonal area of the largest 20\% of fibers was $20.9 \pm 6.6( \pm \mathrm{SD}) \mu \mathrm{m}^{2}$ in the control nerve and $79.5 \pm 43.1( \pm \mathrm{SD}) \mu \mathrm{m}^{2}$ in the axotomized stump (Fig. 6). Neurofilament densities were $640 / \mu \mathrm{m}^{2}$ for swollen fibers and $160 /$ $\mu \mathrm{m}^{2}$ for normal, large fibers. The lengths of these swellings ranged from 50 to several hundred microns. An example of macrophage-mediated myelin clearance around one of the proximal swellings is depicted in Figure 7.

\section{Discussion}

The present studies extend our understanding of axonal biology in two major directions. First, they indicate that even in mammalian peripheral nerves the survival of the axon is not wholly dependent on day-to-day receipt of newly synthesized materials supplied from the cell body. Most of these C57BL/6/Ola mouse axons can conduct action potentials for more than $14 \mathrm{~d}$ (Lunn 

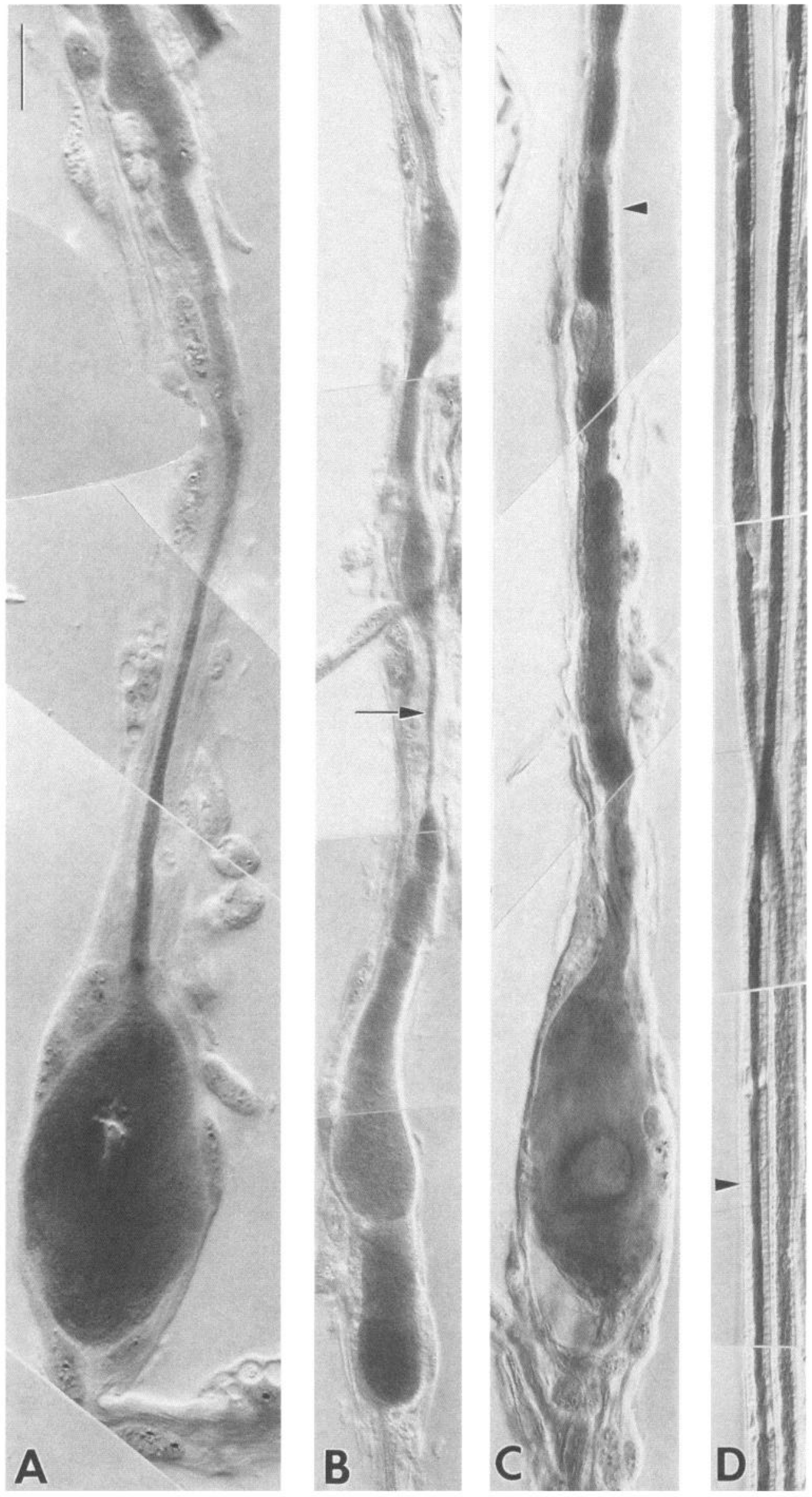

Figure 4. Silver-stained teased fibers from the sciatic distal stump of a C57BL/6/Ola mouse, $14 \mathrm{~d}$ after axotomy $(A-C)$. The site of transection is at the bottom of the figure. Note the massive axonal swellings at the proximal end of these interrupted axons. These silver-positive enlargements are filled with densely packed neurofilaments (cf. Fig. 5). Swelling of the axon, although not as dramatic, is present over long lengths extending distally from the interrupted end. Compare axonal calibers in swollen fibers $(A-C)$ to those in normal fibers $(D)$. A segment of relatively reduced axonal caliber (arrow) is seen in fiber $B$, which likely represents a region with fewer neurofilaments (see Results). Myelin sheaths (arrowheads) are seen around normal fibers and some swollen axonal regions, but the largest areas of swelling are demyelinated. Scale bar, $20 \mu \mathrm{m}$. 

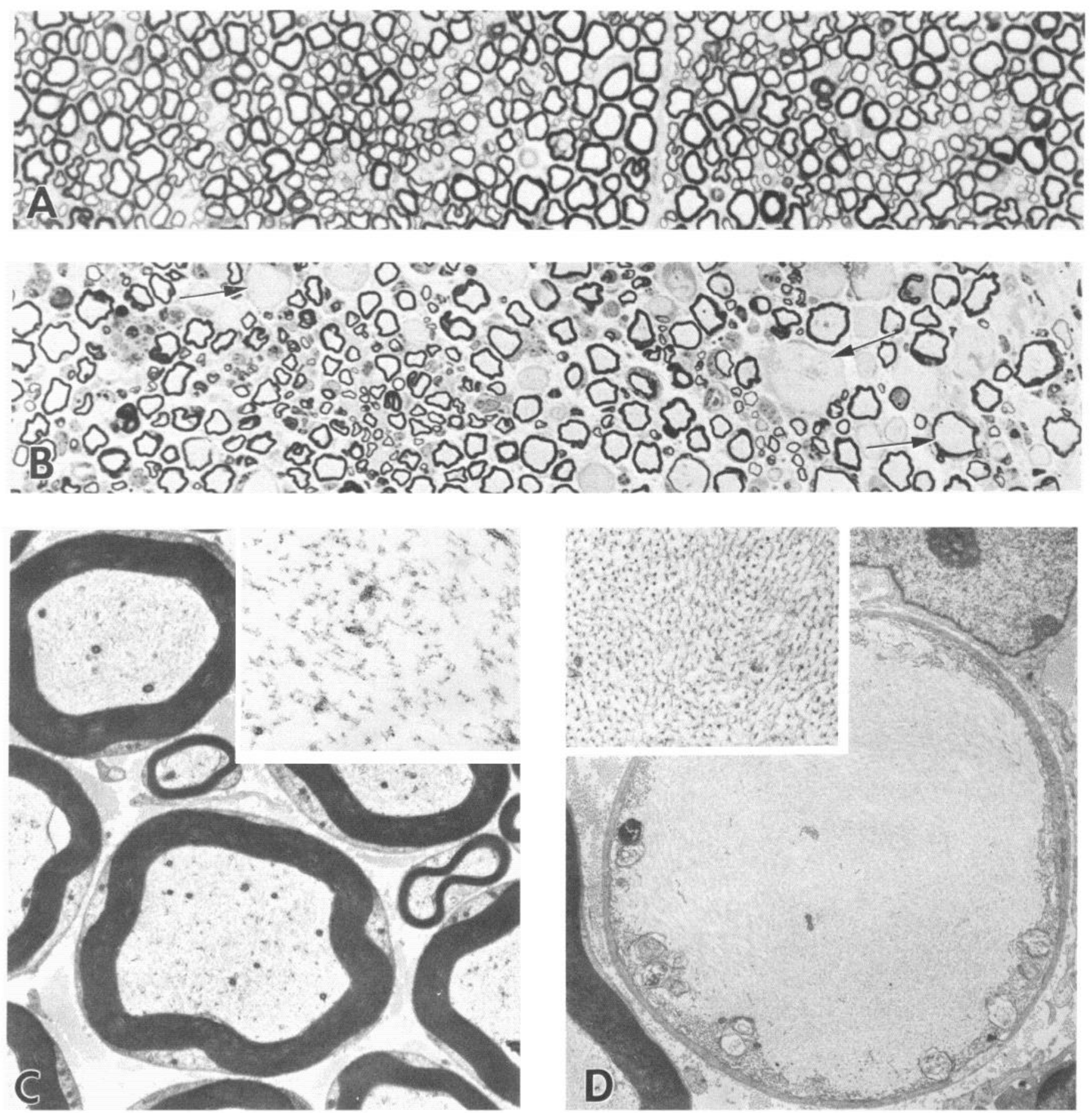

Figure 5. Cross sections of sciatic nerves from C57BL/6/Ola mice that were unoperated $(A$ and $C)$ and axotomized $14 \mathrm{~d}$ previously $(B$ and $D)$. Compare the size and appearance of the normal axons to the swollen axons (arrows) in $1 \mu \mathrm{m}$ plastic sections $(A$ and $B)$ and electron micrographs $(C$ and $D)$. The largest swollen axons have lost their myelin sheaths due to displacement of their normal attachment sites at the paranode (see Results). Insets depict neurofilament densities from representative axons. Stains: $A$ and $B$, toluidine blue; $C$ and $D$, lead citrate and uranyl acetate. Magnification: $A$ and $B, 630 \times ; C$ and $D, 6000 \times$; insets, $44,000 \times$.

et al., 1989), and many survive by morphological criteria for $28 \mathrm{~d}$ while separated from their major source of protein synthesis. Second, the present studies indicate that neurofilaments undergo a slow and progressive redistribution in the distal stump, resulting in accumulations at both the proximal and distal ends of isolated segments. This result suggests that the neurofilaments undergo bidirectional transport in these transected nerves.

\section{Implications of prolonged axonal survival}

The theories accounting for the rapid axonal breakdown usually seen after axotomy have focused on the activation of intrinsic calcium-dependent neutral proteases, which have an affinity for neurofilaments (Schlaepfer and Hasler, 1979; Nixon et al., 1982; Zimmerman and Schlaepfer, 1982). Activation of these prote- 


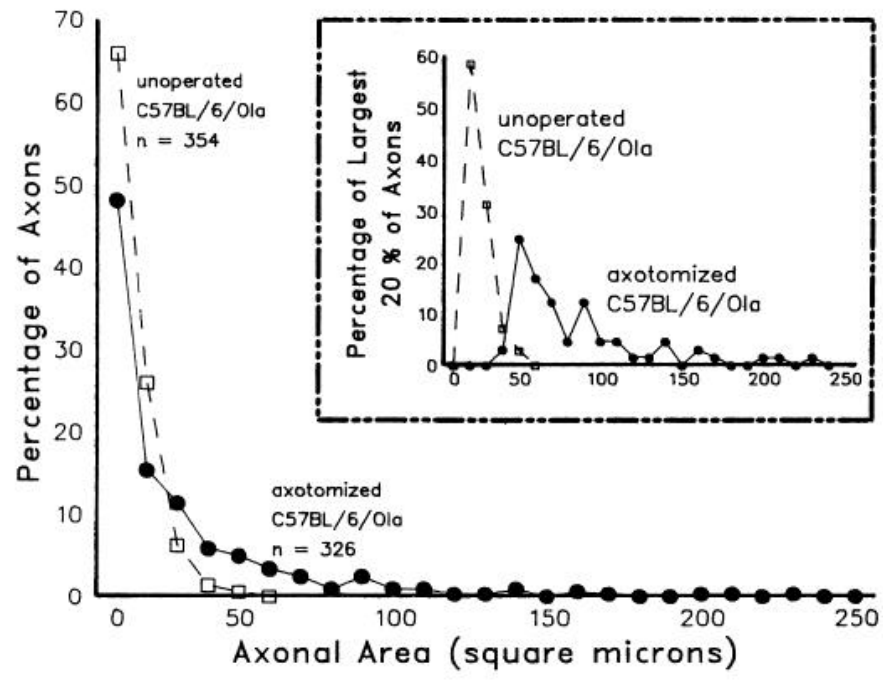

Figure 6. Comparison of axonal areas calculated from the cross sections depicted in Figure 5, $A$ and $B$. Each data point represents a "bin" that includes all axons with cross-sectional areas in a specified range (bin width, $10 \mu \mathrm{m}^{2}$ ). The main graph shows an unselected population of fibers, including all axons in the area sampled. The differences between unoperated and axotomized nerves are better visualized if the largest $20 \%$ of fibers in these samples are compared in the inset graph. Note the substantial difference in the areas of the largest fibers in unoperated versus operated mice.

ases has been thought to be a consequence of interruption of axonal flow from the cell body (Joseph, 1973; Schlaepfer and Bunge, 1973). The absence of rapid disintegration of the axon in the $\mathrm{C} 57 \mathrm{BL} / 6 / \mathrm{Ola}$ mouse suggests that the proteases remain inactive, either because of intrinsic abnormalities or, more likely, because factors other than interruption of proximal to distal flow are necessary for their activation. Recent data demonstrating a defect in the initial recruitment of macrophages in these animals suggest that macrophages may be necessary contributors to the process of rapid axonal breakdown (Perry et al., 1990).

Macrophages have been increasingly recognized as active participants in Wallerian degeneration, being primarily responsible for clearance of myelin (Beuche and Friede, 1984; Stoll et al., 1989) and the regulation of some Schwann cell responses (Lindholm et al., 1987). Systemic administration of anti-macrophage antibodies has been shown to delay the process of Wallerian degeneration in normal rodents (Lunn et al., 1989). Although macrophages were present in increasing numbers as Wallerian degeneration progressed, we found that macrophages infiltrated nerves early at sites of transection and cleared myelin debris while leaving adjacent axons undisturbed (Fig. 7). This indicates that infiltrating macrophages do not randomly phagocytose tissue but are specifically targeted to myelin debris. An attractive hypothesis is that a specific signal is transmitted to macrophages from the injured Schwann cell, activating them to clear myelin. Another distinct signal may be necessary to activate macrophages to initiate axonal breakdown; this signal may be lacking in nerves from C57BL/6/Ola mice.

\section{Lack of regenerative sprouting}

The distal stumps and the isolated segments responded differently from the proximal stumps following peripheral nerve axotomy. In the proximal stump, which remained connected to the cell body, there was a regenerative response (sprouting) at

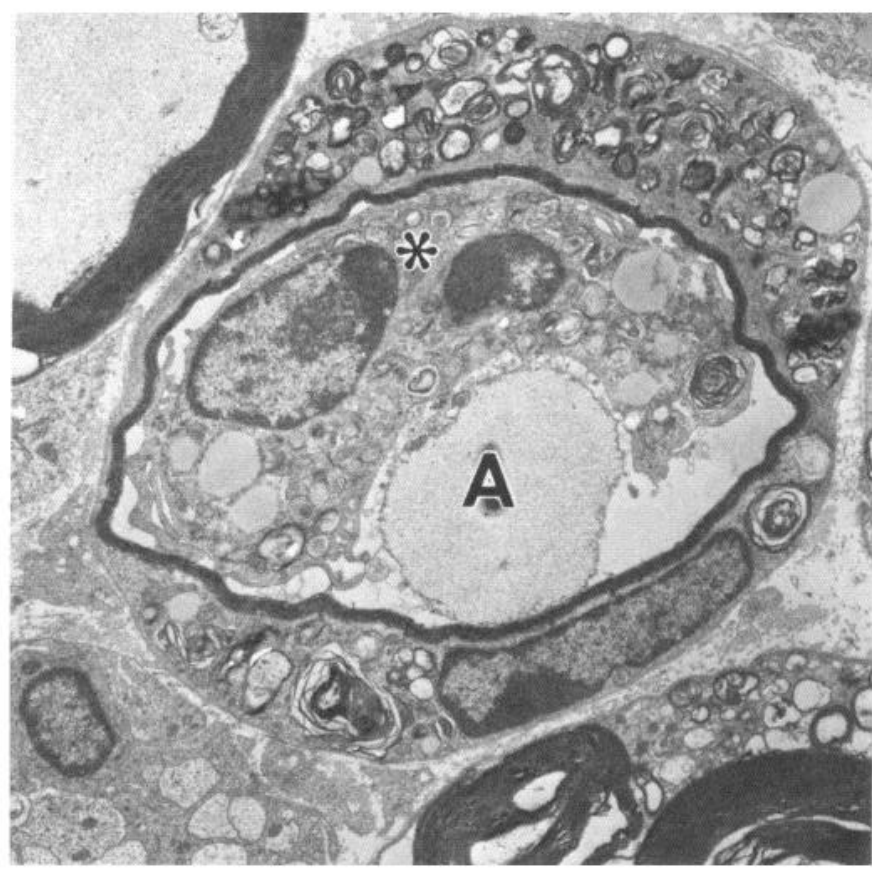

Figure 7. Macrophage (asterisk) within the periaxonal space, digesting a myelin sheath while apparently leaving its axon untouched. This specificity of the macrophage for the damaged sheaths suggests that a distinct signal is necessary to activate macrophages to initiate axonal breakdown. $A$, axon. Stain, lead citrate and uranyl acetate. Magnification, $5200 \times$.

the cut site. In the isolated segments, however, no detectable sprouting occurred at either the distal or proximal faces, indicating that axons separated from their cell bodies do not contain the factors necessary for sprouting. Such factors are likely to include the growth-associated proteins (GAPs; Skene and Willard, 1981; Kalil and Skene, 1986) whose syntheses are upregulated in axotomized neurons (Hoffman, 1989). Studies are underway to test whether axons already containing high levels of GAPs at the time of axotomy might sprout when separated from their cell bodies.

\section{Cytoskeletal reorganization}

The beaded appearance seen on silver stains of axons surviving for at least $7 \mathrm{~d}$ after axotomy represents a redistribution of the cytoskeleton into neurofilament and microtubule domains. A similar appearance is observed when fresh nerve is stretched before freezing (Ochs and Jersild, 1987). This stretch-induced phenomenon is prompt and does not occur in fixed nerves, so it cannot account for the progressive changes described here. The dissociation of neurofilaments and microtubules is borne out by the ultrastructural findings of neurofilamentous swellings containing only a small proportion of microtubules, and shrunken axons composed predominantly of microtubules. Such images, interpreted in concert with the beaded-fiber morphology, are likely to reflect the "moving out" of neurofilaments in relation to microtubules and suggest that at least some of the microtubules may move less or be stationary. In any event, the consequence is the formation of swollen regions that are neurofilament rich and narrowed regions that are neurofilament poor. Judging from the time it takes to occur, this redistribution is most likely an active phenomenon and might use the same mechanism of motility, as yet unknown, that underlies normal slow transport. 
The axonal pathology observed in surviving distal stumps of $\mathrm{C} 57 \mathrm{BL} / 6 / \mathrm{Ola}$ mice bears a remarkable resemblance to that described in human giant axonal neuropathy (Asbury et al., 1972) and in intoxication with 2,5-hexanedione (Spencer and Schaumburg, 1977) and carbon disulfide (Monaco et al., 1985). Experimentally, hexacarbon intoxication is characterized by neurofilamentous swellings, microtubule/neurofilament dissociation, and acceleration of the rate of neurofilament transport (Monaco et al., 1985, 1989; Watson et al., 1991). Similar swellings, although with a different distribution, can be produced by $\beta, \beta^{\prime}-$ iminodipropionitrile (Griffin et al., 1982, 1983a,b; Watson et al., 1989) and 3,4-dimethyl-3,5-hexanedione (Anthony et al., 1983), agents that retard neurofilament transport (Griffin et al., $1978,1984)$. Because these agents contain bifunctional reactive groups, they have been postulated to derivatize covalently the neurofilament subunits, with the resultant adducts acting to "cross-link" neighboring filaments (Anthony et al., 1983). Our data, however, demonstrate that no exogenous neurotoxin is needed to create a very similar pathology, and raise the possibility that some of these toxins might be causing a form of Wallerian degeneration in "slow motion," similar to that seen in the C57BL/6/Ola model.

We detected giant neurofilamentous swellings at proximal faces of distal stumps, or simultaneously at both cut faces of isolated segments, beginning at 3-4 d after transection, and these continued to enlarge to their peak appearance at $14 \mathrm{~d}$. At the distal face, these swellings presumably developed as the result of continued proximal-to-distal transport of neurofilaments to that site. Analogously, giant swellings at the proximal face probably developed from similar active movement of some neurofilaments, but in this case distal to proximal.

Based on conclusions drawn from pulse-labeling experiments, cytoskeletal proteins have becn considered to move only in an anterograde (proximal-to-distal) direction via the slow component of axonal transport (Hoffman and Lasek, 1975). In a nonmammalian system, the garfish, this slow transport of the cytoskeleton is apparent in fibers separated from their cell bodies, where there is a progressive atrophy of the axoplasm that moves from proximal to distal at the rate of slow axonal transport (Cancalon, 1982). In contrast, although our experimental system is analogous to the garfish model, the results demonstrate that mammalian peripheral nerves behave differently when separated from their cell bodies. Instead of the progressive proximal-to-distal axonal atrophy predicted by experiments in the garfish, we observed a structural reorganization characterized by the accumulation of neurofilaments all along the fiber and especially at cut ends. These findings suggest that a proportion of the assembled cytoskeleton in mammalian peripheral nerves may remain stationary and that, at least in axotomized nerves, those neurofilaments that are transported, move bidirectionally. The form in which neurofilaments are transported - soluble subunits or assembled structures-remains unclear.

Two alternative explanations of the neurofilamentous swellings observed at cut sites are that (1) they are merely a consequence of mechanical displacement ("squeezing") of axoplasm by compression at the time of transection (Ochoa et al., 1972), and (2) they are due to "retraction" of the interrupted axon into the distal stump ("retraction balls"). The first can be excluded because the accumulations of neurofilaments developed gradually over weeks. The second is unlikely because nerve crushes produced the same findings as transections, and transected axons did not move farther from the site of interruption with time.
Furthermore, our estimates of the number of neurofilaments within the swellings suggest that all of the neurofilaments normally contained within several millimeters of axonal length would need to be "retracted" or collapsed into a few hundred microns to produce these masses. Therefore, these giant swellings could not develop by retraction of the axoplasm contained within the $200-300 \mu \mathrm{m}$ adjacent to the cut site into a smaller length. By this same argument, we suggest that the accumulated neurofilaments came from a long segment of the distal stump; if they all arose from the region just distal to the neurofilamentous swellings, that region should be depleted of neurofilaments. Because long segments of axons devoid of neurofilaments were not observed, it is likely that some of the neurofilaments in these swellings were contributed from regions even farther from the site of transection.

Whether retrograde transport of neurofilament protein takes place in an unperturbed axon or only occurs in the axon separated from its cell body is an open question. Similarly, these data give no indication of the physical state in which neurofilaments are redistributed; we only know that at the cut sites they accumulate as normal-appearing, although densely packed, neurofilaments. Interestingly, the retrograde transport of neurofilaments or degradation products of neurofilaments has previously been pustulated to provide a feedback or autoregulatory mechanism controlling the synthesis of neurofilament proteins in the cell body (Schlaepfer et al., 1984).

\section{References}

Alpert RM, Grafstein B, Edwards DL (1980) Slow axonal transport in goldfish optic axons. Soc Neurosci Abstr 6:83.

Anthony DC, Boekelheide K, Graham DG (1983) The effect of 3,4dimethyl substitution on the neurotoxicity of 2,5-hexanedione, I. Accelerated clinical neuropathy is accompanied by more proximal axonal swellings. Toxicol Appl Pharmacol 71:362-371.

Asbury AK, Gale MK, Cox SC, Baringer JR, Berg BO (1972) Giant axonal neuropathy: a unique case with segmental neurofilamentous masses. Acta Neuropathol (Berl) 20:237-247.

Beuche W, Friede RL (1984) The role of non-resident cells in Wallerian degeneration. J Neurocytol 13:767-796.

Brimijoin S (1975) Stop-flow: a new technique for measuring axonal transport, and its application to the transport of dopamine-beta-hydroxylase. J Neurobiol 6:379-394.

Brimijoin S, Wiermaa MJ (1977) Rapid axonal transport of tyrosine hydroxylase in rabbit sciatic nerve. Brain Res 120:77-96.

Brown MC, Perry VH, Lunn ER, Gordon S, Heumann R (1991) Macrophage dependence of peripheral sensory nerve regeneration: possible involvement of nerve growth factor. Neuron 6:359-370.

Cancalon P (1982) Slow flow in axons detached from their perikarya. J Cell Biol 95:989-992.

Griffin JW, Price DL, Drachman DB, Engel WK (1976) Axonal transport to and from the motor nerve ending. Ann NY Acad Sci 274:3145.

Griffin JW, Hoffman PN, Clark AW, Carroll PT, Price DL (1978) Slow axonal transport of neurofilament proteins: impairment by $3,3^{\prime}$-iminodipropionitrilc administration. Scicnce 202:633-635.

Griffin JW, Gold BG, Cork LC, Price DL, Lowndes HE (1982) IDPN neuropathy in the cat: coexistence of proximal and distal axonal swellings. Neuropathol Appl Neurobiol 8:351-364.

Griffin JW, Fahnestock KE, Price DL, Hoffman PN (1983a) Microtubule-neurofilament segregation produced by $3,3^{\prime}$-iminodipropionitrile: evidence for association of fast axonal transport with microtubules. J Neurosci 3:557-566.

Griffin JW, Fahnestock KE, Price DL, Cork LC (1983b) Cytoskeletal disorganization induced by local application of 3,3'-iminodipropionitrile and 2,5-hexanedione. Ann Neurol 14:55-61.

Griffin JW, Anthony DC, Fahnestock KE, Hoffman PN, Graham DG (1984) 3,4-Dimethyl-2,5-hexanedione impairs the axonal transport of neurofilament proteins. J Neurosci 4:1516-1526. 
Hoffman PN (1989) Expression of GAP-43, a rapidly transported protein, and class II beta tubulin, a slowly transported protein, are coordinated in regenerating neurons. J Neurosci 9:893-897.

Hoffman PN, Lasek RJ (1975) The slow component of axonal transport: identification of major structural polypeptides of the axon and their generality among mammalian neurons. J Cell Biol 66:351-366.

Hollenbeck PJ (1990) Cytoskclcton on the move. Naturc 343:408409.

Jones HB, Cavanagh JB (1983) Distortions of the nodes of Ranvier from axonal distention by filamentous masses in hexacarbon intoxication. J Neurocytol 12:439-458.

Joseph B (1973) Somatofugal events in Wallerian degeneration: a conceptual overview. Brain Res 59:1-18

Kalil K, Skene JHP (1986) Elevated synthesis of an axonally transported protein correlates with axon outgrowth in normal and injured pyramidal tracts. J Neurosci 6:2563-2570.

Lasek RJ, Hoffman PN (1976) The neuronal cytoskeleton, axonal transport and axonal growth. In: Cell motility, Bk C, Microtubules and related proteins (Goldman R, Pollard T, Rosenbaum J, eds), pp 1021-1051. Cold Spring Harbor, NY: Cold Spring Harbor Laboratory.

Lasek RJ, Garner JA, Brady ST (1984) Axonal transport of the cytoplasmic matrix. J Cell Biol 99:212s-221s.

Lindholm D, Heumann R, Meyer M, Thoenen H (1987) Interleukin-1 regulates synthesis of nerve growth factor in non-neuronal cells of rat sciatic nerve. Nature 330:658-659.

Lubinska L, Niemierko S (1977) Velocity and intensity of bidirectional migration of acetylcholinesterase in transected nerves. Brain Res 27: 329-342.

Lunn ER, Perry VH, Brown MC, Rosen H, Gordon S (1989) Absence of Wallerian degeneration does not hinder regeneration in peripheral nerve. Eur J Neurosci 1:27-33.

Monaco S, Autilio-Gambetti L, Zabel D, Gambetti P (1985) Giant axonal neuropathy: acceleration of neurofilament transport in optic axons. Proc Natl Acad Sci USA 82:920-924.

Monaco S, Jacob J, Jenich H, Patton A, Autilio-Gambetti L, Gambetti $P$ (1989) Axonal transport of neurofilament is accelerated in peripheral nerve during 2,5-hexanedione intoxication. Brain Res 491: 328-334.

Nixon RA, Logvinenko KB (1986) Multiple fates of newly synthesized neurofilament proteins: evidence for a stationary neurofilament network distributed nonuniformly along axons of retinal ganglion cell neurons. J Cell Biol 102:647-659.

Nixon RA, Brown A, Marotta CA (1982) Posttranslational modification of a neurofilament protein during axoplasmic transport: implications for regional specialization of CNS axons. J Cell Biol 94: 150-158.

Ochoa J, Fowler TJ, Gilliatt RW (1972) Anatomical changes in peripheral nerves compressed by a pneumatic tourniquet. J Anat 113: $433-455$.
Ochs S (1982) Mechanisms of transport. In: Axoplasmic transport and its relation to other nerve functions (Ochs S, ed), pp 225-250. New York: Wiley.

Ochs S, Jersild RA (1987) Cytoskeletal organelles and myelin structure of beaded nerve fibers. Neuroscience 22:1041-1056.

Ochs S, Jersild A Jr, Li J-M (1989) Slow transport of freely movable cytoskcletal components shown by beading partition of nerve fibers in the cat. Neuroscience 33:421-430.

Perry VH, Lunn ER, Brown MC, Cahusac S, Gordon S (1990) Evidence that the rate of Wallerian degeneration is controlled by a single autosomal dominant gene. Eur J Neurosci 2:408-413.

Ranish N, Ochs S (1972) Fast axoplasmic transport of acetylcholinesterase in mammalian nerve fibers. J Neurochem 19:2641-2649.

Schlaepfer WW, Bunge RP (1973) The effects of calcium ion concentration on the degradation of amputated axons in tissue culture. J Cell Biol 59:456-470.

Schlaepfer WW, Hasler MB (1979) Characterization of the calciuminduced disruption of neurofilaments in rat peripheral nerves. Brain Res 168:299-309.

Schlaepfer WW, Lee C, Trojanowski JQ, Lee VM-Y (1984) Persistence of immunoreactive neurofilament protein breakdown products in transected rat sciatic nerve. J Neurochem 43:857-864.

Skene JHP, Willard M (1981) Characteristics of growth-associated polypeptides in regenerating toad retinal ganglion cell axons. J Neurosci 1:419-426.

Spencer PS, Schaumburg HH (1977) Ultrastructural studies of the dying back process. III. The evolution of experimental peripheral giant axonal degeneration. J Neuropathol Exp Neurol 36:276-299.

Stanley E, Griffin JW, Fahnestock KE (1985) Effect of IDPN-induced axonal swellings and paranodal demyelination on conduction in motor nerve fibers. J Neurol Sci 69:183-200.

Sternberger LA (1979) Immunocytochemistry. New York: Wiley.

Stoll G, Griffin JW, Li CY, Trapp BD (1989) Wallerian degeneration in the peripheral nervous system: participation of both Schwann cells and macrophages in myelin degradation. J Neurocytol 18:671-683.

Watson DF, Griffin JW, Fittro KP, Hoffman PN (1989) Phosphorylation-dependent immunoreactivity of neurofilaments increases during axonal maturation and IDPN intoxication. J Neurochem 53:18181829.

Watson DF, Fittro KP, Hoffman PN, Griffin JW (1991) Phosphorylation-related immunoreactivity and the rate of transport of neurofilaments in chronic 2,5-hexanedione intoxication. Brain Res 539: 103-109.

Weisenberg RC, Flynn J, Gao B, Awodi S, Skee F, Goodman SR, Riederer BM (1987) Microtubule gelatin-contraction: essential components and relation to slow axonal transport. Science 238:11191121.

Zimmerman U-JP, Schlaepfer WW (1982) Characterization of a brain calcium-activated protease that degrades neurofilament proteins. Biochemistry 21:3977-3983. 